\title{
The Krasnosel'skiǔ-Quittner Formula and Instability of a Reaction-Diffusion System with Unilateral Obstacles
}

\author{
In-Sook Kim and Martin Väth \\ Communicated by Michal Feckan, received April 22, 2014.
}

\begin{abstract}
We prove a formula which relates the fixed point index of a parabolic obstacle equation to a fixed point index related to the right-hand side of the equation. The result is applied to a reaction-diffusion system at a constant equilibrium which is subject to Turing's diffusion-driven instability. It is shown that if a unilateral obstacle is added, the system becomes unstable in a parameter domain where the system without obstacle is stable.
\end{abstract}

\section{Contents}

1. Introduction 230

2. The Krasnoselskii-Quittner Formula 231

3. Instability of the Reaction-Diffusion System 241

4. Other Obstacles

Appendix A. A Local Lipschitz Condition for a Superposition Operator in $L_{p}$ Spaces 248

References

1991 Mathematics Subject Classification. primary 35K87; secondary: 34D20, 35K57, 35K86, 47J20, 47J35, 47H05, 47H11.

Key words and phrases. reaction-diffusion system, Signorini condition, unilateral obstacle, instability, asymptotic stability, Krasnoselskij formula, parabolic obstacle equation.

The paper was written in the framework of the SFB 647 of the DFG and of the Basic Science Research Program through the National Research Foundation of Korea (NRF) funded by the Ministry of Education, Science and Technology (NRF-2012-0008345). Financial support by the DFG and the NRF is gratefully acknowledged. 


\section{Introduction}

For a bounded domain $\Omega \subseteq \mathbb{R}^{N}$ with Lipschitz boundary and $C^{1}$-functions $F_{1}, F_{2}: \mathbb{R}^{2} \rightarrow \mathbb{R}$ consider the reaction-diffusion system

$$
\begin{aligned}
& u_{t}=d_{1} \Delta u+F_{1}(u, v) \\
& v_{t}=d_{2} \Delta v+F_{2}(u, v)
\end{aligned}
$$

with $d_{1}, d_{2}>0$ and Neumann (no-flow) boundary conditions

$$
\frac{\partial u}{\partial n}=\frac{\partial v}{\partial n}=0 \quad \text { on } \partial \Omega .
$$

We assume that $F_{1}, F_{2}$ are such that the system (1), (2) is subject to Turing's "diffusion-driven instability" at some stationary and spatially constant solution $\left(u_{0}, v_{0}\right)$, i.e. in case $d_{1}=d_{2}=0$ the solution is linearly stable. In this case, one can interpret one of $(u, v)$ as the concentration of an activator the other as an inhibitor. We will choose the signs such that $u$ describes the activator.

It is known that $\left(u_{0}, v_{0}\right)$ is then automatically also a linearly stable solution of (1), (2) if either the diffusion speed $d_{1}$ of the activator $u$ is sufficiently large or if the quotient $d_{2} / d_{1}$ is sufficiently small, i.e. if the activator diffuses faster than the inhibitor.

We will prove that if we equip the system with some unilateral obstacle with respect to $v$ at $v_{0}$, e.g. if we replace the Neumann boundary condition for $v$ on some part $\Gamma \subseteq \partial \Omega$ of positive $(N-1)$-dimensional Hausdorff measure by the Signorini condition

$$
v \geq v_{0}, \quad \frac{\partial v}{\partial n} \geq 0, \quad\left(v-v_{0}\right) \frac{\partial v}{\partial n}=0 \quad \text { on } \Gamma,
$$

then (1) fails to be asymptotically stable if $d_{1}$ and $d_{2}$ are both large, independently of the quotient $d_{2} / d_{1}$.

Condition (3) can mean that there is a source for $v$ on $\Gamma$ which becomes active only if the concentration $v$ of the inhibitor would run under the threshold $v_{0}$; the source produces just enough that $v$ does not go under this threshold.

Similar results hold also if the inequalities in (3) are inverted (i.e. if there is a sink which does not let go $v$ over the threshold $v_{0}$ ) or if different type of unilateral obstacles (e.g. in the interior of $\Omega$ or of integral type, or any combinations of such obstacles as long as their direction is the same) are considered.

The main tool of our proof is the Krasnoselskiı-Quittner formula for parabolic variational inequalities which is of independent interest.

The Krasnoselskiı formula for ordinary differential equations relates the fixed point index (local Leray-Schauder mapping degree) of the flow (i.e. of the translation in time map) at a stationary solution $u_{0}$ for small times $t$ with the local mapping degree of the map on the right-hand side of the equation at $u_{0}$. By the homotopy invariance of the fixed point index, one can conclude that if $u_{0}$ is asymptotically stable, then the local index (and thus the local degree) must be 1. In particular, one has a necessary criterion for asymptotic stability (which in case of (1), (3) will be violated for large $d_{1}, d_{2}$, and so we could obtain the announced instability result). However, roughly speaking, the Krasnoselskii formula is known only for ordinary differential equations. 
This is not completely true, since recently the Krasnoselskii formula has been obtained also for (Lipschitz or compact) perturbations for linear generators of semigroups. We remark that just to see that the terms in the Krasnoselskil formula are defined, some compactness requirements are necessary: Either compactness of the semigroup $[\mathbf{3}, \mathbf{5}]$ or at least on the set where the nonlinearity assumes its values [4], or compactness of the nonlinearity $[\mathbf{6}]$ are required.

In the case of variational inequalities the situation is more involved, since the "natural" degree for stationary solutions is actually a fixed point index, and, although the flow is determined by a maximal monotone operator, this operator is only maximal monotone in a different space and hence one cannot directly employ the flow corresponding to that operator as considered e.g. in [19].

Nevertheless, P. Quittner has proved in [14] an instability criterion by showing implicitly in the proof a Krasnoselskil type formula (with the "natural" fixed point index for variational inequalities). Since it is likely that this result was developed completely unaware of the Krasnoselskil formula (although for the proof analogous homotopies as in $[\mathbf{3}, \mathbf{5}]$ are considered), we call the corresponding formula the Krasnoselskiü-Quittner formula.

However, we cannot directly use Quittner's result for the problem (1), (2), because in order to obtain a necessary compactness via smoothness, this result required an assumption which is probably not satisfied for the problem (except in space dimension $N=1$ ). It might be possible to find the required smoothness estimates in certain cases (e.g. if $\partial \Omega$ and $\Gamma \subseteq \partial \Omega$ are "sufficiently nice") by other means, but this would require separate considerations for every type of problem and, e.g. if one considers an unilateral obstacle in an open set $\Omega_{0} \subseteq \Omega$, it seems likely that such a smoothness result would not hold.

For this reason, we go a different approach: By a surprisingly small modification of the arguments in $[\mathbf{1 4}]$, we are actually able to show that the smoothness hypothesis in [14] is completely superfluous. We also relax another hypothesis of [14] and thus are finally able to give a result which in contrast to [14] does neither in its formulation nor in its proof make use of fractional power spaces or fractional power operators.

\section{The Krasnoselskii-Quittner Formula}

If one wants to prove (or even just formulate) the Krasnosel'skiu-Quittner formula for variational inequalities, one faces two difficulties. The first is that a useful formulation of that formula requires that one has a (nonlinear) continuous flow in a fixed state space. The second difficulty is that this flow is required to satisfy some compactness properties so that one can deal with operators whose degree or fixed point index is well-defined.

The former difficulty is related to the fact that a "natural" setting for variational inequalities involves working with a Gel'fand triple $V \subseteq H \subseteq V^{\prime}$ appropriate to the problem, and the naturally associated operator to variational inequalities is (maximal) monotone only in the space $H$ : Hence, at most the space $H$ appears natural to consider the flow. However, the inequality involves a convex set $K \subseteq V$ which typically is closed only in $V$, and so also the metric projection $P_{K}$ onto $K$ is typically only continuous with respect to the topology of $V$. Since a natural formulation of the Krasnosel'ski1-Quittner formula must employ this projection, we are actually forced to formulate it in the space $V$. Fortunately, the situation 
is not so bad: Due to automatic "smoothing" properties, one obtains a (weak) flow in $V$, see e.g. [14, (7)] which is the natural flow for the formulation of the Krasnosel'skiŭ-Quittner formula.

We will see that actually also the compactness of the flow is automatic: At a first glance, this compactness might appear a severe restriction for the cone $K$, and indeed P. Quittner has required in $[\mathbf{1 4}]$ a cumbersome condition which he called (K) in order to obtain compactness of certain operators. We discuss this hypothesis later on.

We will see by a surprisingly simple argument that the hypothesis $(K)$ in $[\mathbf{1 4}$ can be completely omitted, and that actually the compactness of the considered operators is automatic. The difference to $[\mathbf{1 4}]$ is that we do not claim that the compactness is due to the "smoothing" properties of the flow. More precisely, we do not show that the flow assumes values in some fractional power space $X_{\alpha}$ with $\alpha>1 / 2$, but only that it assumes values in $K$. (For this reason, we will discuss later in this section, how one can obtain the smoothness anyway in certain situations).

We are also able to replace another hypothesis of $[\mathbf{1 4}]$ concerning fractional power spaces by a more classical Hölder condition which is actually even a less restrictive requirement, so that finally neither the formulation of our main result nor its proof involves fractional power spaces or fractional power operators at all.

Anyway, since the main part of our proof follows [14], we use the same setting and notation in this section: We assume that $H$ is a real Hilbert space, its scalar product and norm denoted by $(\cdot, \cdot)$ and $|\cdot|$, respectively. The dual space $V^{\prime}$ is understood so that the dual pairing of $V^{\prime}$ and $V$ extends the scalar product $(\cdot, \cdot)$ (in the sense of the embeddings $V \subseteq H \subseteq V^{\prime}$ ), and we use the same symbol for that dual pairing. Moreover, $V$ is assumed to be a real Hilbert space on its own right with inner product $\langle\cdot, \cdot\rangle$ and corresponding norm $\|\cdot\|$. It is assumed that $V$ is a dense subset of $H$, and that the embedding of $V$ into $H$ is compact. The norm in $V^{\prime}$ is the functional norm (with respect to $\|\cdot\|$ ) corresponding to the dual pairing $(\cdot, \cdot)$; it is denoted by $\|\cdot\|_{V^{\prime}}$.

The linear operator $A: H \rightarrow H$ which we consider is defined as the restriction of the linear operator $\mathfrak{A}: V \rightarrow V^{\prime}$,

$$
(\mathfrak{A} u, \varphi)=\langle u, \varphi\rangle \quad \text { for all } u, \varphi \in V,
$$

to the set $D(A):=\mathfrak{A}^{-1}(H) \subseteq V \subseteq H$. In particular, $A: D(A) \rightarrow H$ is a selfadjoint positive operator in $H$. Finally, let $K \subseteq V$ be nonempty, convex and closed in $V$. For a map $F: V \rightarrow H$ (which will later be assumed to satisfy a certain Lipschitz condition on some open set $\mathcal{U} \subseteq V$ ), we are interested in the parabolic type variational inequality

$$
u(t) \in K, \quad\left(\frac{d u(t)}{d t}+\mathfrak{A}(u(t))-F(u(t)), \varphi-u(t)\right) \geq 0 \quad \text { for all } \varphi \in K,
$$

or, equivalently,

(5) $u(t) \in K, \quad\left(\frac{d u(t)}{d t}-F(u(t)), \varphi-u(t)\right)+\langle u(t), \varphi-u(t)\rangle \geq 0 \quad$ for all $\varphi \in K$.

Here, we understand the derivative in the topology of $H$, and as usual we understand by a solution of $(4)$ on $[0, T)$ an absolutely continuous function $u:[0, T) \rightarrow H$ satisfying (5) almost everywhere. However, it is crucial for us that under our later requirements the derivative will exist even for every $t>0$ as a right-sided derivative 
(in $H$ ), and the corresponding formulas (4), (5) hold even for every $t \in(0, T)$, see Lemma 2.

In order to formulate the Krasnosel'skil-Quittner formula for (4) or (5), we have to introduce the metric projection $P_{K}: V \rightarrow K$ which is defined such that for $v \in V$ the image $u=P_{K} v$ is the unique point from $K$ which has closest distance (with respect to $\|\cdot\|)$ to $v$. Since $V$ is a Hilbert space and $K \subseteq V$ is nonempty, closed and convex, it is well-known that $u=P_{K} v$ is uniquely defined by that requirement and characterized by (i.e. the unique solution of) the variational inequality

$$
u \in K, \quad\langle u-v, \varphi-u\rangle \geq 0 \text { for all } \varphi \in K .
$$

Moreover, $P_{K}: V \rightarrow K \subseteq V$ is Lipschitz continuous with constant 1 .

We denote by $\Phi(t)$ the map which associates to every initial value $u_{0} \in K$ the value $u(t)$ where $u$ is a solution of (4) (or (5)) satisfying $u(0)=u_{0}$. Under our hypothesis, it will turn out that $\Phi(t)$ is for $t>0$ a single-valued continuous compact map from (an open subset of) $K$ into $V$, and thus also $\Psi(t):=\Phi(t) \circ P_{K}$ is continuous and compact from (an open subset of) $V$ into $V$.

Recall that if $\mathcal{U} \subseteq V$ is open and bounded and $G: \mathcal{U} \rightarrow V$ is continuous and compact and has exactly one fixed point $u_{0} \in \mathcal{U}$ then one can define the local fixed point index in the space $V$ as

$$
\operatorname{ind}_{V}\left(G, u_{0}\right):=\operatorname{deg}_{V}(i d-G, \mathcal{U}, 0) .
$$

Here, $\operatorname{deg}_{V}$ denotes the Leray-Schauder mapping degree in the space $V$. Recall that the excision property of the degree implies that the same value is obtained if $\mathcal{U}$ is replaced by a smaller open neighborhood of $u_{0}$ so that $\operatorname{ind}_{V}\left(G, u_{0}\right)$ is actually independent of the choice of $\mathcal{U}$ (which justifies the notation).

We call a stationary solution $u_{0}$ of (4) asymptotically stable if for each $\varepsilon>0$ there is $\delta>0$ such that for all $u \in V$ with $\left\|u-u_{0}\right\| \leq \delta$ there holds $\left\|\Phi(t)(u)-u_{0}\right\|<$ $\varepsilon$ for all $t>0$ and $\Phi(t)(u) \rightarrow u_{0}$ as $t \rightarrow \infty$.

Theorem 1. Suppose that $(V,\langle\cdot, \cdot\rangle)$ is dense and compactly embedded into $(H,(\cdot, \cdot))$, and $F: V \rightarrow H$. Let $K \subseteq V$ be closed and convex, and $u_{0}$ be a stationary solution of $(4)$, i.e. $u_{0}=\Phi(t)\left(u_{0}\right)$ for every $t>0$, or, equivalently,

$$
u_{0} \in K, \quad\left(\mathfrak{A}\left(u_{0}\right)-F\left(u_{0}\right), \varphi-u_{0}\right) \geq 0 \quad \text { for all } \varphi \in K .
$$

Suppose that there is an open neighborhood $\mathcal{U} \subseteq V$ of $u_{0}$ such that the restriction $\left.F\right|_{\mathcal{U}}:(\mathcal{U},\|\cdot\|) \rightarrow(H,|\cdot|)$ is Lipschitz continuous and satisfies the following Hölder type condition

$$
\|F(u)-F(v)\|_{V^{\prime}} \leq C|u-v|^{\alpha} \quad \text { for all } u, v \in \mathcal{U},
$$

where $\alpha>0$ and $C \in[0, \infty)$ are independent of $u, v \in \mathcal{U}$. Suppose also that $u \neq P_{K}\left(A^{-1}(F(u))\right.$ for all $u \in \mathcal{U} \backslash\left\{u_{0}\right\}$. Then

$$
\operatorname{ind}_{V}\left(\Phi(t) \circ P_{K}, u_{0}\right)=\operatorname{ind}_{V}\left(P_{K} \circ A^{-1} \circ F, u_{0}\right) \quad \text { for all } t \in\left(0, t_{0}\right] .
$$

If $u_{0}$ is asymptotically stable, then the index in (9) is 1.

REMark 1. Part of the assertion of Theorem 1 is that the fixed point indices in (9) are defined in the sense introduced earlier, i.e., the corresponding operators are compact and have no other fixed points than $u_{0}$ in a neighborhood of $u_{0}$.

Equation (9) is the announced Krasnoselkiu-Quittner formula for variational inequalities. We emphasize that the hypothesis $(\mathrm{K})$ of $[\mathbf{1 4}]$ is completely omitted 
in Theorem 1. Moreover, the hypothesis in the last part of $(\mathrm{F})$ of $[\mathbf{1 4}]$ is replaced by the Hölder condition (8). The condition (8) is not only more convenient to formulate but also strictly weaker as we will show in Remark 5 .

REMARK 2. Our proof of Theorem 1 will show that the hypothesis about the asymptotic stability can be relaxed to the following two assumptions:

(1) There are sequences $0<r_{n} \rightarrow 0$ and $t_{n}>0$ such that for every $u \in K$ with $\left\|u-u_{0}\right\|=r_{n}$ there holds $\left\|\Phi(t)(u)-u_{0}\right\| \leq r_{n}$ for all $t>t_{n}$.

(2) There is no sequence $u_{n} \in K$ with $0<\left\|u_{n}-u_{0}\right\| \rightarrow 0$ such that $u_{n}$ is the initial value of a stationary or periodic solution of (4)

These assumptions are neither weaker nor stronger than the classical notion of Ljapunov stability: They are stronger in the sense that there is no freeness in the coupling of the two uses of $r_{n}$, but they are weaker in the sense that the solutions may go arbitrarily far away from $u_{0}$ if they only return in time.

Since $\Phi$ is completely continuous (as we will show in the proof of Theorem 1), the above two assumptions indeed follow from the asymptotic stability of Theorem 1 with any sequence $r_{n} \rightarrow 0$ (for large $n$ ).

Indeed, there are $\delta_{n} \in\left(0, r_{n}\right)$ such that for every $u \in K$ with $\left\|u-u_{0}\right\|<\delta_{n}$ there holds $\left\|\Phi(t)(u)-u_{0}\right\| \leq r_{n}$ for all $t>0$. We establish the existence of a corresponding $t_{n}$ for every sufficiently large $n$ : For large $n$ we have $\lim _{t \rightarrow \infty} \Phi(t)(u)=0$ for every $u$ from $S:=\left\{u \in K:\left\|u-u_{n}\right\|=r_{n}\right\}$. Since $\Phi$ is continuous, the compact set $C:=\overline{\Phi(1)(S)}$ is covered by finitely many open (in $C$ ) sets $C_{1}, \ldots, C_{k}$ such that there are $s_{1}, \ldots, s_{k}>0$ with $\left\|\Phi\left(s_{j}\right)(u)-u_{0}\right\|<\delta_{n}$ for all $u \in C_{j}(j=1, \ldots, k)$. Since the flow is autonomous, this implies $\left\|\Phi(t)(u)-u_{0}\right\| \leq r_{n}$ for all $t>s_{j}$ and $u \in C_{j}$. Hence, $t_{n}:=1+\max \left\{s_{1}, \ldots, s_{k}\right\}$ has the required property.

Apparently, the reason for considering fractional power spaces in [14] was to obtain compactness of certain auxiliary maps. At a first glance, this is a very natural idea: According to the experience in "classical" parabolic equations, one might expect some regularity of the solution, in particular, some uniform norm estimates in fractional power spaces and hence simple compactness criteria might be expected.

The problem with this idea is that variational inequalities exhibit in general less regularity than classical parabolic equations, and for this reason, the hypothesis (K) of [14] (which actually implies regularity of the solutions) cannot hold in many such situations.

Since we want to avoid such regularity conditions, we must find another compactness result. We prepare this result with some simple observations.

Lemma 1. The operators $\mathfrak{A}: V \rightarrow V^{\prime}$ and $\mathfrak{A}^{-1}: V^{\prime} \rightarrow V$ are norm-preserving bijections, and $A^{-1}$ is compact from $H$ into $V$. Moreover, $\mathfrak{A}^{-1} f \in V$ and $A^{-1} h \in V$ are for $f \in V^{\prime}, h \in H$ characterized by

$$
\left\langle\mathfrak{A}^{-1} f, \varphi\right\rangle=(f, \varphi) \quad \text { and } \quad\left\langle A^{-1} h, \varphi\right\rangle=(h, \varphi) \quad \text { for all } \varphi \in V,
$$

respectively.

Proof. For fixed $f \in V^{\prime}$, the definition of $\mathfrak{A}$ means that $u \in V$ satisfies $\mathfrak{A} u=f$ if and only if $(f, \varphi)=\langle u, \varphi\rangle$ holds for all $\varphi \in V$. By the Riesz' representation theorem, there is exactly one such $u$, and $\|u\|=\|f\|_{V^{\prime}}$. Hence, $\mathfrak{A}: V \rightarrow V^{\prime}$ is a norm-preserving bijection, and we have established the first part of (10). To see the compactness of $A^{-1}: H \rightarrow V$, we note that $A^{-1}=\mathfrak{A}^{-1} \circ j$ where $j: H \rightarrow V^{\prime}$ is 
the embedding described earlier. By hypothesis, $j$ is the Banach-space adjoint of the compact embedding $i: V \rightarrow H$ and thus compact.

Corollary 1. For $h \in H \subseteq V^{\prime}$, we have

$$
\|h\|_{V^{\prime}}=\left\|\mathfrak{A}^{-1} h\right\|=\left\|A^{-1} h\right\| .
$$

In particular, for $\mathcal{U} \subseteq V$ and $F: \mathcal{U} \rightarrow H$ the condition (8) is equivalent to

$$
\left\|A^{-1}(F(u)-F(v))\right\| \leq C|u-v|^{\alpha} \quad \text { for all } u, v \in \mathcal{U} .
$$

The announced compactness result is the following:

Proposition 1. For every $t>0$, every $h \in H \subseteq V^{\prime}$, and every $f \in V^{\prime}$ the variational inequality

$$
u \in K, \quad(t \mathfrak{A} u-h-f, v-u) \geq 0 \quad \text { for all } v \in K
$$

has a unique solution $u=u_{t, h, f}$, and we have

$$
u_{t, h, f}=P_{K}\left(t^{-1}\left(A^{-1} h+\mathfrak{A}^{-1} f\right)\right) .
$$

Moreover, if $t_{0}>0, H_{0} \subseteq H$ is bounded (in $H$ ), and $G \subseteq V^{\prime}$ is relatively compact, then the set

$$
U_{t_{0}, H_{0}, G}:=\left\{u_{t, h, f}: t \geq t_{0}, h \in H_{0}, f \in G\right\}
$$

is relatively compact in $V$.

Proof. By definition of $\mathfrak{A},(12)$ is equivalent to

$$
u \in K, \quad\langle u, v-u\rangle-t^{-1}(h, v-u)-t^{-1}(f, v-u) \geq 0 \quad \text { for all } v \in K .
$$

Using (10), we can rewrite this equivalently as

$$
u \in K, \quad\left\langle u-t^{-1}\left(A^{-1} h+\mathfrak{A}^{-1} f\right), v-u\right\rangle \geq 0 \quad \text { for all } v \in K .
$$

By (6), this in turn is equivalent to (13). This shows the equivalence of (12) and (13) and thus the unique existence of a solution of (12). Now if $t_{0}>0$, $H_{0} \subseteq H$ is bounded and $G \subseteq V^{\prime}$ is relatively compact, then $I_{0}:=\left[0,1 / t_{0}\right], K_{0}:=$ $\overline{A^{-1} H_{0}}$ and $K_{1}:=\mathfrak{A}^{-1}(\bar{G})$ are compact by Lemma 1 . The continuity of the maps $h: I_{0} \times K_{0} \times K_{1} \rightarrow V, h(s, u, v):=s(u+v)$ and $P_{K}$ thus implies that $\bar{U}_{t_{0}, H_{0}, G} \subseteq$ $\left(P_{K} \circ h\right)\left(I_{0} \times K_{0} \times K_{1}\right)$ is compact.

The idea of the proof of Proposition 1 is rather simple: The reason for the compactness of $U_{t_{0}, H_{0}, G}$ is not that its elements are bounded in some "regular" space which is compactly embedded into $V$. Instead, $U_{t_{0}, H_{0}, G}$ is just the continuous image of such a set.

Corollary 2. $u_{0}$ is a stationary solution of (4) if and only if there holds $u_{0}=P_{K}\left(A^{-1} F\left(u_{0}\right)\right)$.

Proof. Since $u_{0}$ is a stationary solution of (4) if and only if (7) holds, we find that this is the case if and only if (12) holds with $h=F\left(u_{0}\right)$ and $f=0$. By Proposition 1 , this is equivalent to $u_{0}=P_{K}\left(A^{-1} h\right)$.

Remark 3. Our hypothesis that $u \neq \Phi(t)(u)$ for all $t \in\left(0, t_{0}\right)$ and all $u \in \mathcal{U} \backslash$ $\left\{u_{0}\right\}$ in Theorem 1 implies that problem (4) has no stationary solutions $u \in \mathcal{U} \backslash\left\{u_{0}\right\}$. By Corollary 2, this is equivalent to $u \neq P_{K}\left(A^{-1} F(u)\right)$ for all $u \in \mathcal{U} \backslash\left\{u_{0}\right\}$. The latter is exactly the hypothesis (D) of [14] which thus is actually automatically satisfied and therefore can be dropped. 
LEMma 2. Let the conditions of Theorem 1 be satisfied with $u_{0}=0$ and $F(0)=$ 0 . Then $u(t)=\Phi(t) v$ exists locally for all $v \in K$ in a neighborhood of 0 and has the following regularity properties.

(1) $u$ is uniformly bounded in $V$ on bounded intervals in $[0, \infty)$, uniformly for $v$ from bounded sets.

(2) The right-sided derivative of $u$ exists (in $H$ ) at every $t>0$, and (4) and (5) hold pointwise when the derivative is understood as this rightsided derivative.

(3) The above right-sided derivative is uniformly bounded in $H$ on bounded intervals in $[0, \infty)$, uniformly for $v$ from bounded sets.

(4) The mapping $(t, v) \mapsto \Phi(t) v$ is compact and uniformly continuous for any set of the form $[c, T] \times(B \cap K)$ where $B \subseteq V$ is a bounded neighborhood of 0 .

Analogous assertions are true if (4) is replaced by

$$
u(t) \in K, \quad\left(\frac{d u(t)}{d t}+\mathfrak{A}(u(t))+F_{0}-F(u(t)), \varphi-u(t)\right) \geq 0 \quad \text { for all } \varphi \in K,
$$

where $F_{0} \in V^{\prime}$ satisfies $\left\langle F_{0}, \varphi\right\rangle \geq 0$ for all $\varphi \in K$.

Proof. This is the main lemma from [14] (with assumptions and conclusions about fractional power spaces omitted). The lemma can be proved as in that paper with the difference that the compactness assertion needs a different argument (since we have no assertions about fractional power spaces available). We obtain this compactness assertion instead from the first three assertions of the lemma and by using Proposition 1. In fact, by the first and third assertion, we obtain that the family

$$
H_{0}=\left\{\frac{d u(t)}{d t}-F(u(t)): u=\Phi(\cdot) v, t \in[c, T], v \in B \cap K\right\}
$$

(the derivative understood as right-sided derivative in $H$ ) is bounded in $H$. By (14) (and by using the second assertion) we can identify the set of points $u(t)=\Phi(t) v$

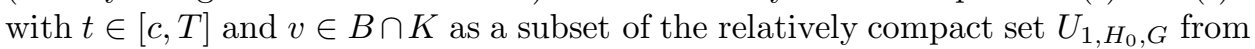
Proposition 1 with $G=\left\{F_{0}\right\}$.

REMARK 4. In view of the last assertion, the additional assumptions $u_{0}=0$ and $F(0)=0$ in Lemma 2 are actually not a loss of generality, since we can just replace $K$ by $\widetilde{K}=K-u_{0}, F$ by $\widetilde{F}(u)=F\left(u-u_{0}\right)-F\left(u_{0}\right)$, and put $F_{0}:=\mathfrak{A} u_{0}+F\left(u_{0}\right)$. Note that $F_{0}=\mathfrak{A} u_{0}-F\left(u_{0}\right)$ satisfies indeed $\left\langle F_{0}, \varphi\right\rangle \geq 0$ for all $\varphi \in \widetilde{K}$ since $u_{0}$ is a stationary solution.

Proof of The First assertion of Theorem 1. Our proof of the first part of Theorem 1 proceeds partially along the line of the proof of the main result in [14]. Therefore, we just sketch the main ideas of the proof, pointing out where we use different arguments.

Step 1. Using the transformations of Remark 4, we discuss only the transformed problem in the following, thus assuming $u_{0}=0, F(0)=0$. Note that we must thus extend the assertion of the theorem for a map $\hat{F}=F-F_{0}$ which assumes values in $H-F_{0} \subseteq V^{\prime}$, and we consider the flow $\Phi_{K}$ generated by (14) instead. 
Complete Continuity of some Resolvent. As in [14], we introduce the auxiliary multivalued operator $A_{K}: K \rightarrow 2^{H}$,

$$
A_{K}(u):=\left\{h \in H:\left(\mathfrak{A} u+F_{0}-h, v-u\right) \geq 0 \text { for all } v \in K\right\} .
$$

We will show that $A_{K}$ has a completely continuous "resolvent". This is a major extension of the arguments of [14]; simultaneously, we simplify the proof.

Proposition 1 implies $A_{K}^{-1}: H \rightarrow K, A_{K}^{-1}(h)=P_{K} \circ\left(A^{-1} h-\mathfrak{A}^{-1} F_{0}\right)=P_{K} \circ$ $\mathfrak{A}^{-1}\left(h-F_{0}\right)$. In particular, $A_{K}^{-1} \circ F=P_{K} \circ \mathfrak{A}^{-1} \circ \hat{F}$. Moreover, for each $\lambda \geq 0, t>0$, we find by applying [20, Theorem 32.C] (with $A=\mathfrak{A}+\lambda t^{-1} i d_{V}, b=t^{-1} h-F_{0} \in V^{\prime}$, $C=K)$ that for each $h \in H$ there is a unique $u \in K$ satisfying $A_{K}(u)=t^{-1}(h-\lambda u)$, that is, the "resolvent" is a map $R_{t, \lambda}:=\left(\lambda i d_{H}+t A_{K}\right)^{-1}: H \rightarrow K$.

We show that the map $R_{0}(t, \lambda, h) \mapsto R_{t, \lambda}(h)$ is continuous and compact into $V$ on each bounded $M \subseteq\left[t_{0}, \infty\right) \times[0, \infty) \times H, t_{0}>0$. Indeed, $u=R_{t, \lambda}(h)$ is equivalent to

$$
u \in K,\left(t \mathfrak{A} u+\lambda u+t F_{0}-h, v-u\right) \geq 0 \text { for all } v \in K .
$$

Fixing some $v \in K$, we thus obtain

$$
\begin{gathered}
t\|u\|^{2} \leq t\|u\|^{2}+\lambda|u|^{2}=(t \mathfrak{A} u+\lambda u, u) \leq \\
-\left(t F_{0}-h, u\right)+t\langle u, v\rangle+\left(\lambda u+t F_{0}-h, v\right) \leq C(1+\|u\|),
\end{gathered}
$$

where $C$ depends only on $M$. It follows that $\|u\|$ is bounded with a constant depending only on $M$. In particular, $h-\lambda u$ are from a bounded subset $H_{0} \subseteq H$. Since $-t F_{0}$ are from a compact subset $G \subseteq V^{\prime}$, we obtain that the set $R_{0}(M)$ of solutions $u$ of (15) is contained in the set $U_{t_{0}, H_{0}, G}$ of Proposition 1 and thus relatively compact in $V$.

Since $\mathfrak{A}: V \rightarrow V^{\prime}$ is continuous, the set of all $(t, \lambda, h, u) \in M \times V$ satisfying (15) is trivially closed in $M \times V$. This means that $R_{0}: M \rightarrow V$ has a closed graph. Since we have already shown that $R_{0}(M)$ is relatively compact in $V$, it follows that $R_{0}: M \rightarrow V$ is continuous, see e.g. [17, Corollary 2.124].

Step 2. We show that for every $t>0$ there holds

$$
\operatorname{ind}_{V}\left(P_{K} \circ \mathfrak{A}^{-1} \circ \hat{F}, 0\right)=\operatorname{ind}_{V}\left(\left(i d_{H}+t A_{K}\right)^{-1} \circ\left(i d_{V}+t F\right) \circ P_{K}, 0\right) .
$$

To this end, we observe first that by the permanence property of the fixed point index, and by our above calculation of $A_{K}^{-1}$, we have

$$
\operatorname{ind}_{V}\left(P_{K} \circ \mathfrak{A}^{-1} \circ \hat{F}, 0\right)=\operatorname{ind}_{V}\left(P_{K} \circ \mathfrak{A}^{-1} \circ \hat{F} \circ P_{K}, 0\right)=\operatorname{ind}_{V}\left(A_{K}^{-1} \circ F \circ P_{K}, 0\right) .
$$

Since $A_{K}^{-1} \circ F=\left(t A_{K}\right)^{-1} \circ(t F)$, it suffices to show by the homotopy invariance of the degree that the map

$$
h_{\lambda}:=\left(\lambda i d_{H}+t A_{K}\right)^{-1} \circ\left(\lambda i d_{V}+t F\right) \circ P_{K}
$$

is an admissible homotopy, that is, there is a neighborhood $U \subseteq V$ of 0 such that $(\lambda, u) \mapsto h_{\lambda}(u)$ is a continuous compact map on $[0,1] \times U$ and such that $h_{\lambda}$ has no fixed points $u \neq 0$ in $U$ for $\lambda \in[0,1]$. Since we have already shown the complete continuity of the resolvent, it remains to prove the latter. However, $u=h_{\lambda}(u)$ is equivalent to $F(u) \in A_{K}(u)$ and thus equivalent to $u=\left(A_{K}^{-1} \circ F\right)(u)=$ $\left(P_{K} \circ \mathfrak{A}^{-1} \circ \hat{F}\right)(u)$. By hypothesis, the latter is excluded for $u \in \mathcal{U} \backslash\{0\}$. 
Step 3. It remains to show that for all sufficiently small $t>0$ there holds

$$
\operatorname{ind}_{V}\left(\left(i d_{H}+t A_{K}\right)^{-1} \circ\left(i d_{V}+t F\right) \circ P_{K}, 0\right)=\operatorname{ind}_{V}\left(\Phi(t) \circ P_{K}, 0\right) .
$$

This is the most technical part of the proof and was done in [14, p. 1173-1175] by showing with a lot of auxiliary estimates that the homotopy

$$
\widetilde{h}_{\lambda}=\left(\lambda\left(i d_{H}+t A_{K}\right)^{-1} \circ\left(i d_{V}+t F\right)+(1-\lambda) \Phi(t)\right) \circ P_{K}
$$

is admissible for all sufficiently small $t>0$. These calculations can be used almost unchanged under our weaker requirements. One only has to be aware that we have relaxed the last condition of $(\mathrm{F})$ from [14] by the weaker Hölder condition (8). However, this last condition of $(\mathrm{F})$ is only used in these calculations to obtain in $[14,(27)]$ estimates of the form

$$
\|\widetilde{F}(u)-\widetilde{F}(v)\| \leq C t^{1 / 2-\beta}
$$

(with $\widetilde{F}:=A^{-1} \circ F$ ) from the estimates

$$
|u-v| \leq \sqrt{t} \text { and }\|u-v\| \leq \widetilde{C} .
$$

We get such estimates already by using even only the first inequality of (16), Indeed, we readily obtain from (11) that

$$
\|\widetilde{F}(u)-\widetilde{F}(v)\| \leq C|u-v|^{\alpha} \leq C t^{\alpha / 2},
$$

and we can proceed as in the proof of [14], only replacing $1 / 2-\beta$ in the exponents throughout by $\alpha / 2$.

Proof of The SECOND ASSERtion of Theorem 1. Let $u_{0}$ satisfy the stability property of Remark 2. This assumption implies that there is some $n$ such that none of the maps $\Phi(t) \circ P_{K}(t>0)$ has a fixed point in the ball $B:=$ $\left\{u \in V:\left\|u-u_{0}\right\|<r_{n}\right\}$ or its closure except $u=u_{0}$. By the homotopy invariance of the degree, we thus obtain that

$$
\operatorname{ind}_{V}\left(\Phi(t) \circ P_{K}, u_{0}\right)=\operatorname{deg}_{V}\left(i d-\Phi(t) \circ P_{K}, B, 0\right)
$$

is the same for every $t>0$, in particular for $t=t_{n}$. Since $\Phi\left(t_{0}\right) \circ P_{K}$ maps $\partial B$ into $B$, the compact homotopy

$$
h(\lambda, u):=(1-\lambda) \Phi\left(t_{0}\right)\left(P_{K}(u)\right)+\lambda u_{0}
$$

shows that the latter degree is $\operatorname{deg}_{V}\left(i d-u_{0}, B, 0\right)$ which is 1 by the normalization property of the degree.

Although, as remarked earlier, neither the formulation nor the proof of Theorem 1 involve fractional power operators or fractional power spaces, the Hölder condition (8) is sometimes easier to verify by using fractional power spaces. So let us briefly introduce these spaces and discuss that condition.

Recall that since $A: D(A) \subseteq H \rightarrow H$ is a selfadjoint positive maximal monotone operator in $H$, one can define the fractional powers $A^{\alpha}$ on their domains $X_{\alpha}=D\left(A^{\alpha}\right)$ which become Hilbert spaces with the corresponding graph norm

$$
\|u\|_{\alpha}:=\left|A^{\alpha} u\right| \text {. }
$$

In particular, $X_{0}=H, X_{1}=D(A)$, and in our setting we have $X_{1 / 2}=V$ by Kato's square root theorem $[\mathbf{1 1}]$. 
REMARK 5. In hypothesis (F) from [14], the following Lipschitz assumption was made instead of (8): There is $\beta<1 / 2$ and $C \in[0, \infty)$ with

$$
\left\|A^{-1}(F(u)-F(v))\right\| \leq C\|u-v\|_{\beta} \quad \text { for all } u, v \in \mathcal{U} \subseteq V \subseteq X_{\beta} .
$$

Note that by Corollary $1,(17)$ is equivalent to

$$
\|F(u)-F(v)\|_{V^{\prime}} \leq C\|u-v\|_{\beta} \quad \text { for all } u, v \in \mathcal{U} \subseteq V \subseteq X_{\beta} .
$$

Our hypothesis (8) is strictly milder than (18). In fact, we need only a Hölder condition of such a type as the following result shows.

Proposition 2. Let $\mathcal{U} \subseteq V$ be bounded, and $F: \mathcal{U} \rightarrow H$. Suppose that there are $\beta<1 / 2, \hat{C} \in[0, \infty)$, and $\hat{\alpha}>0$ with

$$
\|F(u)-F(v)\|_{V^{\prime}} \leq \hat{C}\|u-v\|_{\beta}^{\hat{\alpha}} \quad \text { for all } u, v \in \mathcal{U} \subseteq V \subseteq X_{\beta} .
$$

Then (8) holds with appropriate $C \in[0, \infty)$ and $\alpha>0$.

Proof. The convex interpolation inequality for fractional power spaces (see e.g. [1, Theorem 2.11.1] with $\gamma=0$ and $\alpha=1 / 2$ ) yields

$$
\|u-v\|_{\beta} \leq \widetilde{C}\|u-v\|^{2 \beta}|u-v|^{1-2 \beta} .
$$

Since $\mathcal{U}$ is bounded in $V$ by some constant $M$, we find that (19) implies (8) with $C:=\hat{C}\left(\widetilde{C}(2 M)^{2 \beta}\right)^{\hat{\alpha}}$ and $\alpha:=(1-2 \beta) \hat{\alpha}$.

Although we were able to eliminate the hypothesis (K) of $[\mathbf{1 4}]$ completely from Theorem 1, we shall discuss it now, since it was used in [14] to provide a regularity estimate for (12) and thus for the flow of (4): Such regularity results are of independent interest.

Unfortunately, even the formulation of the hypothesis $(K)$ in $[\mathbf{1 4}]$ is not very clear, since it involves the inverse of a nonlinear map whose existence is unclear. Therefore, we formulate this hypothesis in a simpler manner. Simultaneously, we relax it:

Definition 1. Let $\mathcal{U} \subseteq V, H_{0} \subseteq H, F_{0} \subseteq V^{\prime}, \alpha \in(0,1]$, and $t>0$. We say that $\left(H, V, K, \mathcal{U}, H_{0}, F_{0}, \alpha, t\right)$ satisfy the hypothesis $\left(K^{\prime}\right)$ with respect to the constants $C_{1}, C_{2}, C_{3}, C_{4} \in[0, \infty)$ if $H_{0}$ and $\mathfrak{A}^{-1}\left(F_{0}\right)$ are bounded subsets of $H$ (with constant $C_{1}$ ) and of $X_{(1+\alpha) / 2}$ (with constant $C_{2}$ ), respectively, and if for every $u \in K \cap \mathcal{U}, f \in F_{0}$ and $h \in H_{0}$ there are sequences $v_{n}, g_{n} \in X_{(1+\alpha) / 2}$, $G_{n} \in V$, and $0<\lambda_{n} \rightarrow 0$, such that the following holds for all $n$ :

(1) $u=v_{n}+\lambda_{n} A^{\alpha}\left(v_{n}+g_{n}\right)$.

(2) $v_{n}+\lambda_{n} G_{n} \in K$.

(3) $t\left\langle u, G_{n}\right\rangle-\left(h, G_{n}\right)-\left(f, G_{n}\right) \leq C_{3}$.

(4) $\left\|g_{n}\right\|_{(1+\alpha) / 2} \leq C_{4}$.

If the hypothesis $(\mathrm{K})$ from $[\mathbf{1 4}]$ is satisfied then also the above hypothesis is satisfied for every bounded $H_{0} \subseteq H$ and every $F_{0} \subseteq V^{\prime}$ for which $\mathfrak{A}^{-1}\left(F_{0}\right)$ is a bounded subset of $X_{(1+\alpha) / 2}$. Indeed, in this case we can choose the sequence $v_{n}$ independently of $(u, h, f)$, and put $G_{n}:=0$ and $g_{n}:=G\left(v_{n}\right)$ (with $G$ as in hypothesis $(\mathrm{K})$ of $[\mathbf{1 4}])$. Thus, the hypothesis of Definition 1 is weaker and, in particular, it does not require the existence of nonlinear inverses $\left(i d+\lambda\left(A^{\alpha}+G\right)\right)^{-1}$ as in [14]. Moreover, introducing $G_{n}$ seems to be an important relaxation of the condition in [14]. 
In order to understand the meaning of the condition $v_{n}+\lambda_{n} G_{n} \in K$ in Definition 1 , one should recall that the most interesting case is when $K$ is a cone in $V$ with vertex in $0 \in \mathcal{U}$. In this case, one has $K+K \subseteq K$ and $\lambda K \subseteq K$ for all $\lambda \geq 0$, and thus one can use the following criterion with $K_{0}=K$. However, also for other sets $K$, one usually finds rather large sets $K_{0}$ for which this criterion applies.

Proposition 3. If in Definition 1 there is $K_{0} \subseteq V$ and $\varepsilon>0$ with $(\mathcal{U} \cap K)+$ $\lambda K_{0} \subseteq K$ for all $\lambda \in(0, \varepsilon)$ then the condition $v_{n}+\lambda_{n} G_{n} \in K$ can be replaced by $G_{n}-A^{\alpha}\left(v_{n}+g_{n}\right) \in K_{0}$.

Proof. The choice of $v_{n}$ and $g_{n}$ implies

$$
v_{n}-u=-\lambda_{n} A^{\alpha}\left(v_{n}+g_{n}\right),
$$

and so

$$
v_{n}+\lambda_{n} G_{n}=u+\lambda_{n}\left(G_{n}-A^{\alpha}\left(v_{n}+g_{n}\right)\right) \in(\mathcal{U} \cap K)+\lambda_{n} K_{0} \subseteq K
$$

for all sufficiently large $n$. Hence, one can pass to a subsequence satisfying $v_{n}+$ $\lambda_{n} G_{n} \in K$ for all $n$.

The crucial point of Proposition 3 is that $\lambda_{n}$ does not occur in the condition, and so one can sometimes choose $G_{n}$ (or $\left.-A^{\alpha} g_{n}\right)$ in some "interior" of $K_{0}$ :

ExAmple 1 . Let $\Omega=(a, b) \subseteq \mathbb{R}, H=L_{2}(\Omega), V=W^{1,2}(\Omega)$, and $\alpha<1 / 4$. Then $A^{\alpha}$ is bounded from $V$ into $W^{1-2 \alpha, 2}(\Omega) \subseteq C(\bar{\Omega})$, and thus, putting $\lambda_{n}:=1 / n$ and $g_{n}:=0$, we find for each $u \in \mathcal{U}$ that $v_{n}:=\left(I+\lambda_{n} A^{\alpha}\right)^{-1} u$ has the property that $A^{\alpha}\left(v_{n}+g_{n}\right)=A^{\alpha} v_{n}$ is uniformly small with respect to the max-norm if $\mathcal{U} \subseteq V$ is a sufficiently small neighborhood of 0 . Hence, if $K_{0}$ in Proposition 3 contains a ball in $V$ with respect to the max-norm, we can just let $G_{n}$ be the center of that ball and thus obtain the properties required in Definition 1.

Now we show that the hypothesis $\left(K^{\prime}\right)$ can indeed be used to obtain the following regularity estimate for variational inequalities:

Proposition 4. Let $\left(H, V, K, \mathcal{U}, H_{0}, F_{0}, \alpha, t\right)$ satisfy the hypothesis $\left(K^{\prime}\right)$ of Definition 1 with constants $C_{i}(i=1,2,3,4)$. Let $U_{t, H_{0}, F_{0}}$ denote the set of all solutions of the variational inequality (12) with some $h \in H_{0}, f \in F_{0}$, and $t>0$.

Then $U_{t, H_{0}, F_{0}}$ is a bounded subset of $X_{(1+\alpha) / 2}$. More precisely, a bound for the norm is given by

$$
C_{4}+\left(c_{\alpha} C_{1}+C_{2}\right) / t+\sqrt{\left(c_{\alpha} C_{1} C_{4}+C_{2} C_{4}+C_{3}\right) / t}
$$

where $c_{\alpha}$ is the norm of the embedding of $X_{(1+\alpha) / 2}$ into $X_{\alpha}$.

Proof. With the notation of Definition 1, we note that the positivity of $\mathfrak{A}$ implies

$$
\left(t \mathfrak{A} u, v_{n}-u\right) \leq\left(t \mathfrak{A} v_{n}, v_{n}-u\right) .
$$

Inserting $\varphi:=v_{n}+\lambda_{n} G_{n}$ into (12), we obtain together with (21) that

$0 \leq\left(t \mathfrak{A} u-h-f, v_{n}-u+\lambda_{n} G_{n}\right) \leq\left(t \mathfrak{A} v_{n}-h-f, v_{n}-u\right)+\left(t \mathfrak{A} u-h-f, \lambda_{n} G_{n}\right)$.

Using (20) and dividing by $\lambda_{n}>0$, we conclude with (10) that

$$
\begin{gathered}
0 \leq-\left(t \mathfrak{A} v_{n}-h-f, A^{\alpha}\left(v_{n}+g_{n}\right)\right)+\left(t \mathfrak{A} u-h-f, G_{n}\right)= \\
-t\left(\mathfrak{A} v_{n}, A^{\alpha} v_{n}\right)-t\left(A^{(1+\alpha) / 2} v_{n}, A^{(1+\alpha) / 2} g_{n}\right)+\left(h, A^{\alpha}\left(v_{n}+g_{n}\right)\right) \\
+\left(A^{(1+\alpha) / 2} \mathfrak{A}^{-1} f, A^{(1+\alpha) / 2}\left(v_{n}+g_{n}\right)\right)+\left(t \mathfrak{A} u-h-f, G_{n}\right) .
\end{gathered}
$$


Hence,

$$
\begin{gathered}
t\left\|v_{n}\right\|_{(1+\alpha) / 2}^{2}=\left(t \mathfrak{A} v_{n}, A^{\alpha} v_{n}\right) \leq t\left\|v_{n}\right\|_{(1+\alpha) / 2}\left\|g_{n}\right\|_{(1+\alpha) / 2}+|h|\left\|v_{n}+g_{n}\right\|_{\alpha}+ \\
\left\|\mathfrak{A}^{-1} f\right\|_{(1+\alpha) / 2}\left\|v_{n}+g_{n}\right\|_{(1+\alpha) / 2}+t\left\langle u, G_{n}\right\rangle-\left(h, G_{n}\right)-\left(f, G_{n}\right) \\
\leq t\left\|v_{n}\right\|_{(1+\alpha) / 2} C_{4}+\left(C_{1} c_{\alpha}+C_{2}\right)\left(\left\|v_{n}\right\|_{(1+\alpha) / 2}+C_{4}\right)+C_{3} .
\end{gathered}
$$

Thus, putting $a:=C_{4}+t^{-1}\left(c_{\alpha} C_{1}+C_{2}\right)$ and $b:=t^{-1}\left(c_{\alpha} C_{1} C_{4}+C_{2} C_{4}+C_{3}\right)$, we have shown that $r_{n}:=\left\|v_{n}\right\|_{(1+\alpha) / 2}$ satisfies $r_{n}^{2} \leq a r_{n}+b$. It follows that

$$
\left(r_{n}-\frac{a}{2}\right)^{2} \leq b+\frac{a^{2}}{4} \leq\left(\sqrt{b}+\frac{a}{2}\right)^{2}
$$

and so

$$
\left\|v_{n}\right\|_{(1+\alpha) / 2}=r_{n} \leq C_{0}:=a+\sqrt{b} .
$$

Since $C_{0}$ is independent of $n$, we can assume, passing to a subsequence if necessary, that $v_{n} \rightarrow \widetilde{u}$ in $X_{(1+\alpha) / 2}$. By the compactness of the embedding of $X_{(1+\alpha) / 2}$ into $X_{(1-\alpha) / 2}$, we find that $v_{n} \rightarrow \widetilde{u}$ in $X_{(1-\alpha) / 2}$. Since

$$
\left\|A^{\alpha}\left(v_{n}+g_{n}\right)\right\|_{(1-\alpha) / 2} \leq\left\|v_{n}+g_{n}\right\|_{(1+\alpha) / 2} \leq C_{0}+C_{4}
$$

is bounded, and so (20) implies $v_{n} \rightarrow u$ in $X_{(1-\alpha) / 2}$, we must have $\widetilde{u}=u$. Since the closed ball in $X_{(1+\alpha) / 2}$ with radius $C_{0}$ is weakly closed, we conclude that $\|u\|_{(1+\alpha) / 2} \leq C_{0}$.

\section{Instability of the Reaction-Diffusion System}

Substituting $(u, v)$ by $\left(u-u_{0}, v-v_{0}\right)$ in (1) and (2) or (3), respectively, we can assume without loss of generality that the equilibrium is $u_{0}=v_{0}=0$. Note that after this substitution, $(u, v)$ actually denote the difference to the equilibrium in the original system, so that negative values make physically sense. Linearizing $\left(F_{1}, F_{2}\right)$ at the equilibrium $u_{0}=v_{0}=0$, we rewrite (1) in the form

$$
\begin{aligned}
& u_{t}=d_{1} \Delta u+b_{11} u+b_{12} v+f_{1}(u, v), \\
& v_{t}=d_{2} \Delta v+b_{21} u+b_{22} v+f_{2}(u, v),
\end{aligned}
$$

where $B=\left(b_{i j}\right)_{i j} \in \mathbb{R}^{2 \times 2}$ and

$$
f_{i}(0,0)=0 \text { and } f_{i}^{\prime}(0,0)=0 \quad \text { for } i=1,2 .
$$

We assume that $f_{i}$ satisfy the Lipschitz type condition

$$
\left|f_{i}\left(u_{1}, v_{1}\right)-f_{i}\left(u_{2}, v_{2}\right)\right| \leq C\left(1+\left|u_{1}\right|+\left|u_{2}\right|+\left|v_{1}\right|+\left|v_{2}\right|\right)^{\alpha_{N}}\left(\left|u_{1}-u_{2}\right|+\left|v_{1}-v_{2}\right|\right)
$$

for all $u_{j}, v_{j} \in \mathbb{R}$, where $C \in[0, \infty)$ and $\alpha_{N}:=2 /(N-2)$ if $N>2$ or $0<\alpha_{N}<\infty$ if $N=2$. In case of space dimension $N=1$, we assume instead of (24) only that $f_{i}$ satisfy a local Lipschitz condition in some neighborhood of $(u, v)=(0,0)$.

Note that due to our substitution, we have $v_{0}=0$ so that the corresponding Neumann-Signorini boundary conditions obtain the form

$$
\begin{cases}\frac{\partial u}{\partial n}=0 & \text { on } \partial \Omega, \\ \frac{\partial v}{\partial n}=0 & \text { on } \partial \Omega \backslash \Gamma, \\ v \geq 0, \quad \frac{\partial v}{\partial n} \geq 0, \quad v \frac{\partial v}{\partial n}=0 & \text { on } \Gamma .\end{cases}
$$


We will also assume that $b_{11}>0$ and that the equilibrium $\left(u_{0}, v_{0}\right)=(0,0)$ is for $d_{1}=d_{2}=0$ linearly stable, i.e. the spectrum of $B$ is contained in the left half-plane. This means

$$
b_{11}>0, \quad b_{11}+b_{22}<0, \quad|B|:=b_{11} b_{22}-b_{12} b_{21}>0 .
$$

Note that (26) automatically implies

$$
b_{11}>0>b_{22}, \quad b_{12} b_{21}<0 .
$$

In particular, depending on the sign of $b_{12}$, system (22) is of activator-inhibitor or substrate-depletion type.

By Turing's effect of "diffusion-driven instability" [15], it may happen that the system loses stability in case $d_{1}, d_{2}>0$. More precisely, this is well-known for Neumann (no-flow) boundary conditions (2): In this case, the right-hand side of system (22), (2) is differentiable, and by considering the linearization, one can exactly determine those $\left(d_{1}, d_{2}\right)$ for which the trivial solution of the system is linearly stable: Let $0=\kappa_{0}<\kappa_{1}<\kappa_{2}<\ldots$ denote the eigenvalues of $-\Delta$ with respect to Neumann boundary conditions $(2)$. To each positive eigenvalue $\kappa_{n}(n=1,2, \ldots)$ we associate the hyperbola

$$
\begin{aligned}
C_{n} & =\left\{\left(d_{1}, d_{2}\right) \in \mathbb{R}_{+}^{2}:\left(\kappa_{n} d_{1}-b_{11}\right)\left(\kappa_{n} d_{2}-b_{22}\right)=b_{12} b_{21}\right\} \\
& =\left\{\left(d_{1}, d_{2}\right) \in \mathbb{R}_{+}^{2}: d_{2}=\frac{b_{12} b_{21} / \kappa_{n}^{2}}{d_{1}-b_{11} / \kappa_{n}}+\frac{b_{22}}{\kappa_{n}}\right\},
\end{aligned}
$$

see Figure 1.

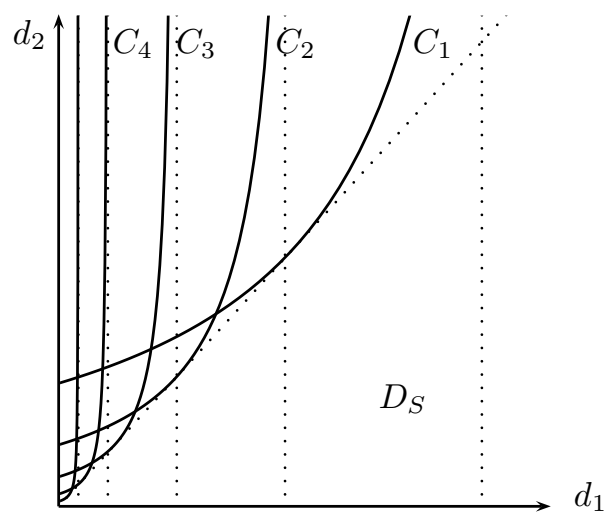

FiguRE 1. Hyperbolas (27) determining $D_{S}$, their vertical asymptotes, and the common tangential line with slope (28)

By standard arguments, it can be verified that Figure 1 is qualitatively correct in all cases with (26): $C_{n}$ is a connected subset of one "branch" of a hyperbola, and $C_{n}$ has the vertical asymptote $b_{11} / \kappa_{n}$. Moreover, the family $C_{n}$ has the common tangent which is a line through $(0,0)$ with slope

$$
S:=\frac{-b_{12} b_{21}+|B|+2 \sqrt{-b_{12} b_{21}|B|}}{b_{11}^{2}}>1 .
$$


One can show that the trivial solution of (22), (2) is linearly stable if and only if $d=\left(d_{1}, d_{2}\right)$ lies to the right/under the common envelope of the hyperbolas $C_{1}, C_{2}, \ldots$; we denote this "domain of stability" by $D_{S}$, see Figure 1. Roughly speaking, by "crossing" the hyperbola $C_{n}$, one loses the corresponding multiplicity of $\kappa_{n}$ of "stable directions". In space dimension $N=1$ this was shown in [13], for $N>1$ see e.g. [7]; a more precise variant of the assertion, showing the asymptotic and exponential stability for $d \in D_{S}$ in the $W^{1,2}(\Omega)$-topology, is given in [18].

In particular, the trivial solution of (22), (2) is linearly stable if the diffusion speeds $d_{1}$ and $d_{2}$ corresponding to the activator $u$ and inhibitor $v$, respectively, satisfy

$$
d_{1} \geq \frac{b_{11}}{\kappa_{1}} \quad \text { or } \quad \frac{d_{2}}{d_{1}}<S
$$

Thus, roughly speaking, each of these inequalities will actually prevent Turing's diffusion-driven instability in (22), (2). In practice, especially the second inequality is often satisfied since the slope (28) is typically extremely large.

In [12], it was shown that if one has a unilateral obstacle like e.g. (25) (recall that $v_{0}=0$ after our substitution) on some part $\Gamma \subseteq \partial \Omega$ of positive measure, there occurs a bifurcation of stationary solutions in $D_{S}$ if $d_{1}, d_{2}$ are both large and, surprisingly, the ratio $d_{2} / d_{1}$ is small. In the following, we will combine a part of the proof of [12] with the Krasnosel'ski1-Quittner formula to prove that, with a unilateral obstacle, the system (2) fails to be asymptotically stable at $\left(u_{0}, v_{0}\right)=(0,0)$ at least if the diffusion speeds $d_{1}$ and $d_{2}$ are both sufficiently large, independently of the ratio $d_{2} / d_{1}$. In space dimension $N=1$, we can make even more quantitative statements by using the results from [10].

In order to apply the Krasnosel'skil-Quittner formula, we first reformulate our problem in a weak sense: We let

$$
K_{0}:=\left\{v \in W^{1,2}(\Omega):\left.v\right|_{\Gamma} \geq 0\right\}
$$

where the restriction $\left.v\right|_{\Gamma}$ is understood in the sense of traces. Let $K:=W^{1,2}(\Omega) \times$ $K_{0}$. Then the weak formulation of problem (22), (25) can be written as

$$
\begin{gathered}
\left(\begin{array}{l}
u \\
v
\end{array}\right) \in K, \quad \text { and for all }\left(\begin{array}{l}
\varphi \\
\psi
\end{array}\right) \in K: \\
\int_{\Omega}\left(u_{t}-b_{11} u-b_{12} v-f_{1}(u, v)\right)(\varphi-u) d x+d_{1} \int_{\Omega} \nabla u(\nabla \varphi-\nabla u) d x \geq 0, \\
\int_{\Omega}\left(v_{t}-b_{21} u-b_{22} v-f_{2}(u, v)\right)(\psi-v) d x+d_{2} \int_{\Omega} \nabla v(\nabla \psi-\nabla v) d x \geq 0 .
\end{gathered}
$$

To rewrite (30) in the setting of Section 2 , we equip $V_{0}:=W^{1,2}(\Omega)$ with the usual scalar product

$$
\langle u, v\rangle:=\int_{\Omega} u(x) v(x) d x+\int_{\Omega} \nabla u(x) \cdot \nabla v(x) d x .
$$

It will be convenient to define $d_{0}:=1$. For $i=0,1,2$, we equip $H_{i}:=L_{2}(\Omega)$ with the scaled scalar product

$$
(u, v)_{i}=d_{i}^{-1} \int_{\Omega} u(x) v(x) d x \quad(i=0,1,2)
$$


(i.e. $H_{0}=L_{2}(\Omega)$ with the usual scalar product), and we define $\mathfrak{A}_{i}: V_{0} \rightarrow V_{0}^{\prime}$ by the duality

$$
\left(\mathfrak{A}_{i} u, v\right)_{i}=\langle u, v\rangle \quad(i=0,1,2) .
$$

We let $A_{i}$ denote the restriction of $\mathfrak{A}_{i}$ to $\mathfrak{A}_{i}^{-1}\left(H_{i}\right)$. Then

$$
\mathfrak{A}_{i}=d_{i} \mathfrak{A}_{0} \quad \text { and } \quad A_{i}=d_{i} A_{0} \quad \text { for } i=1,2 .
$$

We equip $V:=V_{0} \times V_{0}$ and $H:=H_{1} \times H_{2}$ with the canonical inherited scalar products. Then we have in the notation of Section 2 that

$$
\mathfrak{A}\left(\left(\begin{array}{l}
u \\
v
\end{array}\right)\right)=\left(\begin{array}{l}
\mathfrak{A}_{1} u \\
\mathfrak{A}_{2} v
\end{array}\right)=\left(\begin{array}{l}
d_{1} \mathfrak{A}_{0} u \\
d_{2} \mathfrak{A}_{0} v
\end{array}\right)
$$

and

$$
A\left(\left(\begin{array}{l}
u \\
v
\end{array}\right)\right)=\left(\begin{array}{l}
A_{1} u \\
A_{2} v
\end{array}\right)=\left(\begin{array}{l}
d_{1} A_{0} u \\
d_{2} A_{0} v
\end{array}\right) .
$$

By Lemma 1, we find that $A^{-1}$ is compact from $H$ into $V$ and is characterized by

$$
\left\langle A^{-1}\left(\left(\begin{array}{l}
u \\
v
\end{array}\right)\right),\left(\begin{array}{l}
\varphi \\
\psi
\end{array}\right)\right\rangle=\left(\left(\begin{array}{l}
u \\
v
\end{array}\right),\left(\begin{array}{l}
\varphi \\
\psi
\end{array}\right)\right) \quad \text { for all }\left(\begin{array}{l}
\varphi \\
\psi
\end{array}\right) \in V .
$$

Since

$$
A^{-1}\left(\left(\begin{array}{l}
u \\
v
\end{array}\right)\right)=\left(\begin{array}{l}
d_{1}^{-1} A_{0}^{-1} u \\
d_{2}^{-1} A_{0}^{-1} v
\end{array}\right)
$$

we find that $A_{0}^{-1}$ is compact from $H_{0}$ into $V_{0}$, and characterized by

$$
\left\langle A_{0}^{-1} u, v\right\rangle=(u, v)_{0}=\int_{\Omega} u(x) v(x) d x \quad \text { for all } u, v \in H_{0} .
$$

In particular, the restriction $\widehat{A}:=\left.A_{0}^{-1}\right|_{V_{0}}: V_{0} \rightarrow V_{0}$ is compact and characterized by

$$
\langle\widehat{A} u, v\rangle=(u, v)_{0}=\int_{\Omega} u(x) v(x) d x \quad \text { for all } u, v \in V_{0} .
$$

Finally, for $i=1,2$, we define superposition operators $F_{i}: V \rightarrow H_{0}$ by

$$
F_{i}(u, v)(x):=f_{i}(u(x), v(x)),
$$

and $F: V \rightarrow H$ by

$$
F\left(\left(\begin{array}{l}
u \\
v
\end{array}\right)\right):=\left(\begin{array}{l}
d_{1} u+b_{11} u+b_{12} v+F_{1}(u, v) \\
d_{2} v+b_{21} u+b_{22} v+F_{2}(u, v)
\end{array}\right)
$$

Then (30) can be written in the form (5), i.e. in the form (4): Note that if

$$
U(t)(x)=\left(\begin{array}{l}
u(t, x) \\
v(t, x)
\end{array}\right)
$$

is absolutely continuous in the sense that it can be written as the Bochner integral in $H$ (or $V$ ) over its derivative then by $H \cong L_{2}\left(\Omega, \mathbb{R}^{2}\right)$, we can interpret this derivative indeed as

$$
\frac{d U(t)}{d t}(x)=\left(u_{t}(t, x), v_{t}(t, x)\right)
$$

almost everywhere with the partial derivatives $u_{t}$ and $v_{t}$ existing almost everywhere, see e.g. [16, Theorem 4.4.4]. 
REMARK 6. Except in space dimension $N=1$, it is probably not the case that the hypothesis $\left(K^{\prime}\right)$ of Definition 1 can be satisfied with our above choice of $K$. Although it is probably possible to obtain conclusions similar to that of Proposition 4 anyway (i.e. even without hypothesis $\left(K^{\prime}\right)$ ) for this particular cone $K$ by using regularity theory for Signorini problems, the crucial point of our Theorem 1 (in contrast to [14]) is that we do not need such a regularity theory for the Krasnosel'skii-Quittner formula or for the instability result. In fact, in Section 4, we will discuss a similar setting for which one cannot even expect such regularity results.

Proposition 5. There is a neighborhood $\mathcal{U} \subseteq V$ of 0 such that the restriction $\left.F\right|_{\mathcal{U}}:(\mathcal{U},\|\cdot\|) \rightarrow(H,|\cdot|)$ is Lipschitz continuous and satisfies (8). In case $N>2$, this is even true for every bounded $\mathcal{U} \subseteq V$.

Proof. We assume first $N \geq 2$. Let $p^{*}:=2\left(\alpha_{N}+1\right)$ and $\left(p^{*}\right)^{\prime}:=p^{*} /\left(p^{*}-\right.$ 1). Then the Sobolev embedding theorems yield continuous embeddings $V \subseteq$ $L_{p^{*}}\left(\Omega, \mathbb{R}^{2}\right)$, and thus also $L_{\left(p^{*}\right)^{\prime}}\left(\Omega, \mathbb{R}^{2}\right) \subseteq V^{\prime}$, and moreover, for every $p<p^{*}$ there is $\beta<1 / 2$ with $X_{\beta} \subseteq L_{p}\left(\Omega, \mathbb{R}^{2}\right)$. Note that $p^{*}>2$ and thus $\left(p^{*}\right)^{\prime}<2$. From Proposition 6 in the appendix, we thus obtain that $F: L_{p^{*}}\left(\Omega, \mathbb{R}^{2}\right) \rightarrow L_{2}\left(\Omega, \mathbb{R}^{2}\right)$ satisfies a Lipschitz condition on bounded subsets and, if $p<p^{*}$ was chosen sufficiently close to $p^{*}$, then also $F: L_{p}\left(\Omega, \mathbb{R}^{2}\right) \rightarrow L_{\left(p^{*}\right)^{\prime}}\left(\Omega, \mathbb{R}^{2}\right)$ satisfies a Lipschitz condition on bounded subsets. Summarizing, if $\mathcal{U} \subseteq V$ is bounded then $\mathcal{U} \subseteq L_{p^{*}}\left(\Omega, \mathbb{R}^{2}\right) \subseteq$ $L_{p}\left(\Omega, \mathbb{R}^{2}\right)$ is bounded, and $\left.F\right|_{\mathcal{U}}:(\mathcal{U},\|\cdot\|) \rightarrow(H,|\cdot|)$ and $\left.F\right|_{\mathcal{U}}:\left(\mathcal{U},\|\cdot\|_{\beta}\right) \rightarrow V^{\prime}$ are Lipschitz. The latter means (18) and thus implies (8) by Proposition 2.

In case $N=1$, we even have continuous embeddings $V, X_{\beta} \subset C\left(\Omega, \mathbb{R}^{2}\right)$ (if $\beta<1 / 2$ is sufficiently close to $1 / 2)$. The local Lipschitz condition of $f_{i}$ in a neighborhood of $(0,0)$ implies that there is a neighborhood $\mathcal{U}_{0} \subseteq C\left(\Omega, \mathbb{R}^{2}\right)$ of 0 such that $F: \mathcal{U}_{0} \rightarrow C\left(\Omega, \mathbb{R}^{2}\right)$ satisfies a Lipschitz condition on $\mathcal{U}_{0}$. Let $\mathcal{U} \subseteq V$ be a neighborhood of 0 which is contained in $\mathcal{U}_{0}$. Then $\left.F\right|_{\mathcal{U}}: \mathcal{U} \rightarrow C\left(\Omega, \mathbb{R}^{2}\right) \subseteq H$ and $\left.F\right|_{\mathcal{U}}:\left(\mathcal{U},\|\cdot\|_{\beta}\right) \rightarrow C\left(\Omega, \mathbb{R}^{2}\right) \subseteq H \subseteq V^{\prime}$ satisfy a Lipschitz condition on $\mathcal{U}$. From the latter it follows as above by Proposition 2 that (8) holds.

We call $\left(d_{1,0}, d_{2,0}\right)$ a non-bifurcation point of stationary solutions of problem (22), (25) if there is a neighborhood of $\left(d_{1,0}, d_{2,0}, 0,0\right)$ in $\mathbb{R}^{2} \times V^{2}=\mathbb{R}^{2} \times$ $W^{1,2}(\Omega)^{2}$ such that every stationary weak solution $\left(d_{1}, d_{2}, u, v\right)$ in this neighborhood satisfies $u=v=0$. Now we can formulate our main result:

TheOREM 2. Let $\Omega \subseteq \mathbb{R}^{N}$ be a bounded domain with Lipschitz boundary, and suppose (26). Let $\Gamma \subseteq \partial \Omega$ have positive measure. Then there are constants $d_{1,0}, d_{2,0}>0$ such that for every $f_{1}, f_{2}: \mathbb{R}^{2} \rightarrow \mathbb{R}$ satisfying (23) and (24) the following holds.

The set $D \subseteq \mathbb{R}_{+}^{2}$ of non-bifurcation points of stationary solutions of problem (22), (25) is open and contains $\left[d_{1,0}, \infty\right) \times\left[d_{2,0}, \infty\right)$. Let $D_{\infty}$ denote the connected component of $D$ containing $\left(d_{1,0}, d_{2,0}\right)$, in particular $\left[d_{1,0}, \infty\right) \times\left[d_{2,0}, \infty\right) \subseteq$ $D_{\infty}$. Then for every $\left(d_{1}, d_{2}\right) \in D_{\infty}$ the trivial solution of problem (22), (25) fails to be asymptotically stable with respect to the topology of $W^{1,2}(\Omega)$.

In case of space dimension $N=1, \Omega=(a, b)$, every choice $d_{2,0}>d_{*}$, in case $\Gamma=\partial \Omega=\{a, b\}$ even $d_{2,0}>d_{*} / 4$, is possible where $d_{*}$ is the unique positive solution of the equation

$$
\frac{1}{b-a} \sqrt{\frac{d_{*}}{-b_{22}}} \tanh \left((b-a) \sqrt{\frac{-b_{22}}{d_{*}}}\right)=\frac{|B|}{-b_{12} b_{21}} .
$$


Moreover, in case of space dimension $N=1$, there is some $d_{1,1}>0$ (independent of $\left.f_{1}, f_{2}\right)$ such that $D_{\infty}$ contains all $\left(d_{1}, d_{2}\right) \in D_{S}$ with $d_{1} \geq d_{1,1}$.

All above assertions hold unchanged if both inequality signs in (25) are reversed.

REMARK 7. The trivial solution not only fails to be asymptotically stable but even fails to be stable in the sense described in Remark 2 .

Proof. The fact that $D$ is open follows immediately from the definition. That it contains some $\left[d_{1,0}, \infty\right) \times\left[d_{2,0}, \infty\right)$ has been shown in $[\mathbf{1 2}]$, and the corresponding assertions about $D_{\infty}$ in space dimension $N=1$ are contained in [10]. For $i=1,2$, we put $\widetilde{F}_{i}:=d_{i}^{-1} A_{0}^{-1} \circ F_{i}$. By (31), we find that $\widetilde{F}_{i}: V \rightarrow V_{0}$ is characterized by

$$
\left\langle\widetilde{F}_{i}(u, v), \varphi\right\rangle=\int_{\Omega} d_{i}^{-1} f_{i}(u(x), v(x)) \varphi(x) d x \quad \text { for all } \varphi \in V_{0} .
$$

Hence, defining $G: V \rightarrow V$ by

$$
G\left(\left(\begin{array}{l}
u \\
v
\end{array}\right)\right):=\left(\begin{array}{l}
\frac{b_{11}+d_{1}}{d_{1}} \widehat{A} u+b_{12} \widehat{A} v+\widetilde{F}_{1}(u, v) \\
b_{21} \widehat{A} u+\frac{b_{22}+d_{2}}{d_{2}} \widehat{A} v+\widetilde{F}_{2}(u, v)
\end{array}\right),
$$

we are exactly in the situation of [12] where it has been shown that for $\left(d_{1}, d_{2}\right) \in$ $\left[d_{1,0}, \infty\right) \times\left[d_{2,0}, \infty\right)$ the map $P_{K} \circ G$ has an isolated zero at $(0,0)$ and satisfies

$$
\operatorname{ind}_{V}\left(P_{K} \circ G, 0\right)=0 \text {. }
$$

Since $D$ is open and thus locally path-connected, it follows straightforwardly from the homotopy invariance of the degree and the definition of $D$ that the index from (32) is defined for all $\left(d_{1}, d_{2}\right) \in D$ and depends locally constant from $\left(d_{1}, d_{2}\right) \in$ $D$. In particular, $(32)$ holds even for all $\left(d_{1}, d_{2}\right) \in D_{\infty}$. Note now that $G=A^{-1} \circ F$, and so

$$
\operatorname{ind}_{V}\left(P_{K} \circ A^{-1} \circ F, 0\right)=0 \neq 1
$$

for all $\left(d_{1}, d_{2}\right) \in D_{\infty}$. Thus, the assertion follows from the second part of Theorem 1 .

\section{Other Obstacles}

Let $\Omega \subseteq \mathbb{R}^{N}$ be bounded with a Lipschitz boundary, and $\Omega_{0} \subseteq \Omega$ open and satisfy $\bar{\Omega}_{0} \subseteq \Omega$. We consider the problem

$$
\begin{gathered}
u_{t}=d_{1} \Delta u+b_{11} u+b_{12} v+f_{1}(u, v) \quad \text { on } \Omega, \\
v_{t}=d_{2} \Delta v+b_{21} u+b_{22} v+f_{2}(u, v) \quad \text { on } \Omega \backslash \Omega_{0}, \\
-v_{t}+d_{2} \Delta v+b_{21} u+b_{22} v+f_{2}(u, v) \leq 0 \leq v \quad \text { on } \Omega_{0}, \\
\left(-v_{t}+d_{2} \Delta v+b_{21} u+b_{22} v+f_{2}(u, v)\right) v=0 \quad \text { on } \Omega_{0}
\end{gathered}
$$

with Neumann boundary conditions (2).

The last two lines in (33) can mean that there is a source in $\Omega_{0}$ guaranteeing that $v \geq 0$ by becoming just enough active in those points $x$ for which $v(x)$ would become negative without the source.

The weak formulation of (33), (2) has the form (30) when we define $K:=$ $W^{1,2}(\Omega) \times K_{0}$ with

$$
K_{0}:=\left\{v \in W^{1,2}(\Omega):\left.v\right|_{\Omega_{0}} \geq 0\right\} .
$$

In this weak formulation, we can even allow arbitrary $\Omega_{0} \subseteq \bar{\Omega}$, but if $\bar{\Omega}_{0}$ touches the boundary, then in the classical interpretation of (30), one has to replace the Neumann condition (2) for $v$ by the Signorini condition (3) with $\Gamma:=\overline{\Omega_{0}} \cap \partial \Omega$. 
Similarly, we actually do not have to assume that $\Omega_{0}$ is open, if we understand $\left.v\right|_{\Omega_{0}}$ in the sense of traces.

We call $\left(d_{1,0}, d_{2,0}\right)$ a non-bifurcation point of stationary solutions of (30) if there is a neighborhood of $\left(d_{1,0}, d_{2,0}, 0,0\right)$ in $\mathbb{R}^{2} \times W^{1,2}(\Omega)^{2}$ such that every nontrivial stationary solution $\left(d_{1}, d_{2}, u, v\right)$ in this neighborhood satisfies $u=v=0$.

The hypothesis $-1 \notin K_{0}$ of the following theorem is satisfied if $\Omega_{0}$ has positive $N$-dimensional Lebesgue measure. However, it can also be satisfied for certain "thin" obstacles $\Omega_{0}$ (of positive Hausdorff dimension $N-1$ ) if one understands the restriction $\left.v\right|_{\Omega_{0}}$ in the sense of a corresponding trace. The function $u_{-}$of the following result exists e.g. whenever $\Omega \backslash \Omega_{0}$ has an interior point.

TheOREM 3. Let $\Omega \subseteq \mathbb{R}^{N}$ be bounded with a Lipschitz boundary, and $\Omega_{0}$ as above. Assume that $-1 \notin K_{0}$ and that there is $u_{-} \in K_{0}$ with $\int_{\Omega} u_{-}(x) d x<0$. Suppose (26). Then there are constants $d_{i, 0}>0$ such that for every $f_{i}: \mathbb{R}^{2} \rightarrow \mathbb{R}$ satisfying (23) and (24) the following holds.

The set $D \subseteq \mathbb{R}_{+}^{2}$ of non-bifurcation points is open and contains $\left[d_{1,0}, \infty\right) \times$ $\left[d_{2,0}, \infty\right)$. Let $D_{\infty}$ denote the connected component of $D$ containing $\left(d_{1,0}, d_{2,0}\right)$, in particular $\left[d_{1,0}, \infty\right) \times\left[d_{2,0}, \infty\right) \subseteq D_{\infty}$. Then for every $\left(d_{1}, d_{2}\right) \in D_{\infty}$ the trivial solution of problem (30) fails to be asymptotically stable with respect to the topology of $W^{1,2}(\Omega)$.

All above assertions hold unchanged if all inequality signs are reversed and if the hypothesis $-1 \notin K_{0}$ is replaced by the hypothesis $1 \notin K_{0}$.

REMARK 8. The trivial solution not only fails to be asymptotically stable but even fails to be stable in the sense described in Remark 2 .

Proof. The proof is completely analogous to the proof of Theorem 2; the condition $\emptyset \neq \Omega_{0} \neq \Omega$ is used in Lemma 3.1 of $[\mathbf{1 2}]$.

Note that the condition about the measure of $\Omega_{0}$ from [12] is not needed in our case, since this condition was only used in [12] to obtain that $\operatorname{ind}_{V}\left(P_{K} \circ G, 0\right)=1$ if $d_{1}$ is large and $d_{2}$ is sufficiently small. Without this condition, we thus cannot claim that there is a branch of bifurcation points for the stationary problem in $D_{S}$, but nevertheless we obtain the instability result. We conjecture that a reason for this can be that if $\Omega_{0}$ has sufficiently large volume then it can happen that $(u, v)=(0,0)$ is unstable for every $\left(d_{1}, d_{2}\right) \in D_{S}$, and thus there cannot be stationary nontrivial solutions close to $(0,0)$.

We point out that in contrast to the Signorini problem (22), (25), one cannot expect strong regularity results for the solutions of Problem (33), (2). In particular, one probably cannot expect that the solutions of this problem belong to some fractional power space $X_{\alpha}$ with $\alpha>1 / 2$ for $t>0$, and so the way of [14] to prove the compactness of the flow is not possible. This is one of the reasons why it is so important that we could get rid of the condition (K) of [14].

We obtain results analogous to Theorem 3 when we put

$$
K_{0}:=\left\{v \in W^{1,2}(\Omega): \int_{\Gamma_{i}} v d x \geq 0 \text { for } i=1, \ldots, n\right\}
$$

or

$$
K_{0}:=\left\{v \in W^{1,2}(\Omega): \int_{\Omega_{i}} v d x \geq 0 \text { for } i=1, \ldots, m\right\}
$$


where $\Gamma_{1}, \ldots, \Gamma_{n} \subseteq \partial \Omega$ or $\Omega_{1}, \ldots, \Omega_{m} \subseteq \bar{\Omega}$ have pairwise disjoint closure. In this case (and if $\Omega_{i}$ are open with $\bar{\Omega}_{i} \subseteq \Omega$ ), Problem (30) can be interpreted as the weak formulation of (22) with integral Signorini conditions

(34) $\begin{cases}\frac{\partial u}{\partial n}=0 & \text { on } \partial \Omega, \\ \frac{\partial v}{\partial n}=0 & \text { on } \partial \Omega \backslash\left(\Gamma_{1} \cup \cdots \cup \Gamma_{n}\right), \\ \int_{\Gamma_{i}} v d x \geq 0, \quad \frac{\partial v}{\partial n}=\text { const } \geq 0, \quad \int_{\Gamma_{i}} v d x \frac{\partial v}{\partial n}=0 & \text { on } \Gamma_{i}\end{cases}$

or as the weak formulation of the integral obstacle system

$$
\begin{array}{cl}
u_{t}=d_{1} \Delta u+b_{11} u+b_{12} v+f_{1}(u, v) \quad \text { on } \Omega \\
v_{t}=d_{2} \Delta v+b_{21} u+b_{22} v+f_{2}(u, v) \quad \text { on } \Omega \backslash\left(\Omega_{1} \cup \cdots \cup \Omega_{m}\right), \\
-v_{t}+d_{2} \Delta v+b_{21} u+b_{22} v+f_{2}(u, v)=\text { const } \leq 0, \int_{\Omega_{i}} v d x \geq 0 \quad \text { on } \Omega_{i}, \\
\left(-v_{t}+d_{2} \Delta v+b_{21} u+b_{22} v+f_{2}(u, v)\right) \int_{\Omega_{i}} v d x=0 \quad \text { on } \Omega_{i}
\end{array}
$$

with Neumann boundary conditions (2), respectively, see e.g. [8, Observation 5.2] or $[\mathbf{9}$, Appendix], respectively.

Of course, it is also possible to reverse all corresponding inequalities or to combine various of the above obstacles by considering the intersection of the corresponding cones $K_{0}$. However, our proof breaks down if we would attempt to combine obstacles of opposite sign: The proof strongly relies on the fact that there is a constant function $e$ satisfying $e \in K_{0} \backslash\left(-K_{0}\right)$. The special role of the constant function was explained in [12]: It comes from the fact that this is the unique eigenfunction of $\hat{A}$ to its largest eigenvalue.

\section{Appendix A. A Local Lipschitz Condition for a Superposition Operator in $L_{p}$ Spaces}

Proposition 6. Let $\Omega$ be a measure space, and $E_{0}, E_{1}$ be Banach spaces. Let $1 \leq q \leq p<\infty$, and $f: \Omega \times E_{0} \rightarrow E_{1}$ be such that $f(\cdot, u)$ is (strongly Bochner) measurable for every $u \in E_{0}$, and $f(\cdot, 0) \in L_{q}\left(\Omega, E_{1}\right)$.

Suppose that there are $C \in[0, \infty)$ and $a \in L_{p}(\Omega,[0, \infty])$ such that, for almost all $x \in \Omega$,

(36) $\|f(x, u)-f(x, v)\| \leq C(a(x)+\|u\|+\|v\|)^{(p / q)-1}\|u-v\| \quad$ for all $u, v \in E_{0}$.

Then $F(u)(x):=f(x, u(x))$ acts from $L_{p}\left(\Omega, E_{0}\right)$ into $L_{q}\left(\Omega, E_{1}\right)$ and satisfies a Lipschitz condition on every bounded set $U \subseteq L_{p}\left(\Omega, E_{0}\right)$.

In the scalar case $E_{0}=E_{1}=\mathbb{R}$ and if $\Omega$ has no atoms and finite measure, it follows from [2, Theorem 3.10] in view of [2, Theorem 3.1] that the conditions given in Proposition 6 are necessary and sufficient for the conclusion. However, for us the vector-valued version is important. In this case, we do not know whether the condition (36) is really necessary, but it seems at least close to necessary, even if one is only interested in a Lipschitz condition on some neighborhood of 0 .

Proof. It follows from e.g. [16, Theorem A.1.1] and the continuity of $f(x, \cdot)$ for almost all $x \in \Omega$ that $F(u)$ is measurable for measurable $u: \Omega \rightarrow E_{0}$. Similarly, also

$$
G(u, v)(x):=C(a(x)+\|u(x)\|+\|v(x)\|)^{(p / q)-1}
$$


and

$$
H(u, v)(x):=\|u(x)-v(x)\|
$$

are measurable if $u, v: \Omega \rightarrow E_{0}$ are measurable. Let $|\cdot|_{p}$ denote the norm in $L_{p}$. Since

$$
\|F(u)(x)-F(v)(x)\| \leq r(u, v)(x):=G(u, v)(x) H(u, v)(x)
$$

and $F(0) \in L_{q}\left(\Omega, E_{1}\right)$, we thus obtain the result from the estimate

$$
|r(u, v)|_{q} \leq L_{u, v}|H(u, v)|_{p}=L_{u, v}|u-v|_{p},
$$

which follows from Hölder's inequality with

$$
\begin{gathered}
L_{u, v}:=|G(u, v)|_{p q /(p-q)}=C|a(\cdot)+\|u(\cdot)\|+\|v(\cdot)\||_{p}^{(p / q)-1} \\
\leq C\left(|a|_{p}+2 R\right)^{(p / q)-1}
\end{gathered}
$$

for $|u|_{p},|v|_{p} \leq R$

Note that $L_{u, v}$ in the above proof of Proposition 6 is actually an upper bound for the Lipschitz constant.

\section{References}

[1] Amann, H. (1995). Linear and quasilinear parabolic problems, vol. I, Birkhäuser, Basel, Boston, Berlin.

[2] Appell, J. and Zabreı̆ko, P. P. (1990). Nonlinear superposition operators, Cambridge Univ. Press, Cambridge.

[3] Ćwiszewski, A. (2006). Topological degree methods for perturbations of operators generating compact $C_{0}$ semigroups, J. Differential Equations 220 (2), 454-477.

[4] (2011). Periodic oscillations for strongly damped hyperbolic beam equation, Topol. Methods Nonlinear Anal. 37 (2), 259-282.

[5] _ (2011). Positive periodic solutions of parabolic evolution problems: A translation along trajectories approach, Central European J. of Math. 9 (2), 244-268.

[6] Ćwiszewski, A. and Kokocki, P. (2008). Krasnosel'skǐ type formula and translation along trajectories method for evolution equations, Discrete Contin. Dynam. Systems 22 (3), 605628.

[7] Drábek, P., Kučera, M., and Míková, M. (1985). Bifurcation points of reaction-diffusion systems with unilateral conditions, Czechoslovak Math. J. 35, 639-660.

[8] Eisner, J., Kučera, M., and Recke, L., (2002). Smooth continuation of solutions and eigenvalues for variational inequalities based on the implicit function theorem, J. Math. Anal. Appl. 274, 159-180.

[9] Eisner, J., Kučera, M., and Väth, M. (2010). Bifurcation points for a reaction-diffusion system with two inequalities, J. Math. Anal. Appl. 365, 176-194.

[10] Eisner, J. and Väth, M. (2011). Location of bifurcation points for a reaction-diffusion system with Neumann-Signorini conditions, Advanced Nonlinear Studies 11, 809-836.

[11] Kato, T. (1961). Fractional powers of dissipative operators, J. Math. Soc. Japan 13, 246-274.

[12] Kučera, M. and Väth, M. (2012). Bifurcation for a reaction-diffusion system with unilateral and Neumann boundary conditions, J. Differential Equations 252, 2951-2982.

[13] Mimura, M., Nishiura, Y., and Yamaguti, M. (1979). Some diffusive prey and predator systems and their bifurcation problems, Ann. N. Y. Acad. Sci. 316, 490-510.

[14] Quittner, P. (1990). An instability criterion for variational inequalities, Nonlinear Anal. 15 (12), 1167-1180.

[15] Turing, A. M. (1952). The chemical basis of morphogenesis, Phil. Trans. R. Soc. London Ser. B 237 (641), 37-72.

[16] Väth, M. (1997). Ideal spaces, Lect. Notes Math., no. 1664, Springer, Berlin, Heidelberg.

[17] (2012). Topological analysis. From the basics to the triple degree for nonlinear Fredholm inclusions, de Gruyter, Berlin, New York. 
[18] - (2013). Instability of Turing type for a reaction-diffusion system with unilateral obstacles modeled by variational inequalities, Math. Bohem., Proceedings of Equadiff 13, in press.

[19] Vrabie, I. I. (1987). Compactness methods for nonlinear evolutions, 2nd ed., Pitman Publ., Oxford.

[20] Zeidler, E. (1990). Nonlinear functional analysis and its applications, vol. II/A, Springer, New York, Berlin, Heidelberg.

In-Sook Kim, Department of Mathematics, Sungkyunkwan University, Suwon 440746, KOREA

E-mail address: iskim@skku.edu

Martin Väth, Free University of Berlin, Math. Institute, Arnimallee 3, D-14195 Berlin, Germany

E-mail address: vaeth@mathematik.uni-wuerzburg.de 